\title{
NGS reveals relevant resistance mutations
}

Sanger sequencing is the gold-standard method for detection of mutations in $B C R-A B L 1$ in patients with chronic myeloid leukaemia (CML) undergoing treatment with BCR-ABL1 tyrosine-kinase inhibitors (TKIs), an approach recommended in most clinical practice guidelines. Now, the results of the NEXT-in-CML study provide a rationale for incorporating next-generation sequencing (NGS) into the guidelines.

In this multicentre study, the performance of NGS-based screening of loss-of-function $B C R-A B L 1$ mutations was assessed prospectively in consecutive patients with CML and a 'failure' $(n=124)$ or 'warning' $(n=112)$ response to TKI treatment, defined according to the 2013 European LeukemiaNet criteria. $B C R-A B L 1$ mutations were detected using Sanger sequencing and NGS in 60 and 111 patients ( $25 \%$ and $47 \%$ ), respectively. Among 231 patients followed up, the detection of mutations informed decisions such as a change to another TKI $(n=85)$, allogeneic stem cell transplantation $(n=7)$ and an increased frequency of monitoring $(n=21)$.
Mutations detected using NGS only were referred to as low-level mutations. These mutations were related to resistance to imatinib or second-generation BCR-ABL1 TKIs in 69 of 80 patients. On-treatment monitoring demonstrated that the burden of low-level resistance mutations accumulates in patients who continue to receive or are switched to a TKI that is inactive against their specific mutation. Indeed, all of the 21 patients with a warning response whose treatment was not changed despite harbouring low-level resistance mutations subsequently had a failure response.

In summary, these results indicate that the clones that will ultimately result in drug resistance and drive disease progression are not always detectable using Sanger sequencing. The use of NGS as a new gold standard for monitoring patients with CML receiving $B C R-A B L 1$ TKIs is worth considering.

Diana Romero

ORIGINAL ARTICLE Soverini, S. et al. Prospective assessment of NGS-detectable mutations in CML patients with non-optimal response: the NEXT-in-CML study. Blood https://doi.org/10.1182/blood.2019002969 (2019)

\section{Dasatinib versus imatinib in paediatric ALL}

3-4\% of paediatric acute lymphoblastic leukaemias are Philadelphia chromosome-positive $\left(\mathrm{Ph}^{+} \mathrm{ALL}\right)$ and thus express oncogenic BCR-ABL1 kinase that is sensitive to imatinib. Accordingly, imatinib has greatly improved the outcomes of children with $\mathrm{Ph}^{+} \mathrm{ALL}$, but resistance and relapse is common. Now, a phase III trial has revealed that superior outcomes can be achieved with the second-generation BCR-ABL1 inhibitor dasatinib.

In this trial, children with $\mathrm{Ph}^{+} \mathrm{ALL}$ were randomly assigned to receive dasatinib $(n=92)$ or imatinib $(n=97)$ during frontline induction, consolidation and continuation chemotherapy. Randomization was stopped after a planned interim analysis showed a significant improvement in event-free survival (EFS), the primary end point. At 4 years, EFS was $71.0 \%$ with dasatinib and $48.9 \%$ with imatinib (HR 2.36, 95\% Cl 1.27-4.40; $P=0.007$ ). Moreover, 4-year overall survival was $88.4 \%$ and $69.2 \%$, respectively (HR 2.26, 95\% Cl 1.02-4.99; $P=0.04)$. Notably, the 4-year risk of isolated central nervous system (CNS) relapse was lower with dasatinib ( $2.7 \%$ versus $8.4 \% ; P=0.06$ ).

In previous studies of imatinib, EFS was $\sim 60 \%$ at 5 years, but almost all patients received prophylactic cranial irradiation $(\mathrm{PCl})$ and most underwent allogeneic stem cell transplantation, both of which can have serious and long-term adverse effects. In the current trial, no patient received $\mathrm{PCl}$ and only four underwent transplantation.

Both treatments were well tolerated and had a similar profile of toxicities, most commonly infections, pancreatitis and seizures. Five fatal infections occurred in each group, but were not directly attributed to dasatinib or imatinib treatment.

Together, these findings suggest that the ability of dasatinib to penetrate the CNS and to inhibit mutant forms of BCR-ABL1 that are resistant to imatinib enables improved outcomes, without excess toxicity and whilst avoiding interventions with potentially deleterious effects, which is important in paediatric patients.

\section{David Killock}

ORIGINAL ARTICLE Shen, S. et al. Effect of dasatinib vs imatinib in the treatment of pediatric Philadelphia chromosome-positive acute lymphoblastic leukemia a randomized clinical trial.JAMA Oncol. https://doi.org/ 10.1001/jamaoncol.2019.5868 (2020)

\section{SCREENING}

\section{NELSON updated}

Lung cancer has a high incidence worldwide and thus, population-based screening programmes have been proposed as a feasible approach to improve the outcomes of patients with this disease. Now, the publication of 10-year follow-up results of the NELSON trial further informs this debate.

On the basis of a questionnaire sent to $\sim 600,000$ individuals of $50-74$ years of age, 15,792 respondents with a history of smoking were randomly assigned (1:1) to be part of the screening group (four rounds of low-dose CT screening for lung cancer at baseline, 1,3 and 5.5 years) or to the control group (no screening). Data on randomization date, sex, date of lung-cancer diagnosis, and date and cause of death were obtained after approximately 5, 7 and 10-11 years of follow up. Among male participants, 6,583 were assigned to the screening group and 6,612 to the control group. The average screening uptake was $90.0 \%$, with 22,600 CT scans performed $(9.2 \%$ of which needed to be repeated). A positive result was obtained in 467 CT scans (2.1\%), subsequently resulting in 203 screening-detected lung cancers.

At 10 years of follow up, the cumulative incidence of lung cancer was 5.58 and 4.91 cases per 1,000 person-years ( 344 and 304 lung cancers) in the screening and control group, respectively (rate ratio $1.14,95 \% \mathrm{Cl}$ $0.97-1.33$ ) and thus, $59.0 \%$ of cancers detected in the screening group were screeningdetected cancers. These cancers were more often diagnosed at stage IA-B (58.6\%) than non-screening-detected cancers in the screening group (14.2\%) and cancers detected in the control group (13.5\%). The frequency of diagnosis of stage IV lung cancers was $9.4 \%$, $51.8 \%$ and $45.7 \%$, respectively. Cumulative mortality from lung cancer was 2.50 and 3.30 deaths per 1,000 person-years (156 and 206 deaths) in the screening and control group, respectively (rate ratio $0.76,95 \% \mathrm{Cl}$ 0.61-0.94). All-cause mortality was 13.93 and 13.76 deaths per 1000 person-years, respectively.

Overall, these results suggest that population-based CT screening can increase the likelihood of detecting lung cancers at an early stage. Therefore, together with optimal treatment, this approach could improve the chance of long-term survival.

Diana Romero

ORIGINAL ARTICLE de Koning, H. J. et al. Reduced lungcancer mortality with volume $\mathrm{CT}$ screening in a randomized trial. N. Engl.J. Med. 382, 503-513 (2020) 